\title{
ALIMENTACIÓN Y POBREZA POR CARENCIA DE ACCESO A LOS ALIMENTOS EN POBLACIÓN DEL NORESTE DE MÉXICO
}

\author{
FOOD AND POVERTY DUE TO LACK OF FOOD ACCESS IN THE POPULATION OF NORTHEAST MEXICO \\ Ramos Peña Esteban Gilberto *, Salas García Rogelio *, Núñez Rocha Georgina Mayela*, Ramírez \\ López Erik*
}

*Universidad Autónoma de Nuevo León (UANL), México.

\begin{abstract}
Citation: Ramos Peña EG., Salas GR., Núñez Rocha GM., Ramírez López E., (2018) Alimentación y pobreza por carencia de acceso a los alimentos en población del noreste de México. Revista de Salud Pública y Nutrición, 17(2), 11-17.

Editor: Esteban G. Ramos Peña, Dr. CS., Universidad Autónoma de Nuevo León, Facultad de Salud Pública, Monterrey Nuevo León, México.

Copyright: (C2018 Ramos Peña EG et al. This is an open-access article distributed under the terms of Creative Commons Attribution License [CC BY 4.0], which permits unrestricted use, distribution, and reproduction in any medium, provided the original author and source are credited.

Competing interests: The authors have declared that no competing interests exist.

DOI: https://doi.org/10.29105/respyn17.2-2
\end{abstract}

Recibido: 26 de abril 2018;

Aceptado: 20 de junio 2018

Email: esteban.ramosp@uanl.mx 


\title{
ALIMENTACIÓN Y POBREZA POR CARENCIA DE ACCESO A LOS ALIMENTOS EN POBLACIÓN DEL NORESTE DE MÉXICO
}

\author{
Ramos Peña Esteban Gilberto *, Salas García Rogelio *, Núñez Rocha Georgina Mayela*, Ramírez López Erik* \\ *Universidad Autónoma de Nuevo León (UANL), México.
}

\section{RESUMEN}

Introducción: La alimentación es el factor primordial que afecta a la salud, la calidad del patrón de consumo de alimentos puede ser modificada por la pobreza por acceso a los alimentos. Objetivo: Estimar si la calidad de la alimentación está asociada a la pobreza por acceso a los alimentos en población del noreste de México. Material y Métodos: Se analizó la base de datos de la Encuesta Estatal de Salud y Nutrición Nuevo León 2011/2012 que contiene una muestra probabilística de 7,290 individuos. La variable calidad del patrón de consumo alimentario se construyó a partir de la Encuesta Estatal de Salud y Nutrición Nuevo León $2011 / 2012$ y la variable pobreza por acceso a los alimentos a partir de registros nacionales. Se utilizaron pruebas estadísticas no paramétricas. Resultados: El mayor porcentaje de la población analizada se encuentra en la categoría de necesidad de cambio en la calidad del patrón de consumo alimentario, el cuartil con menor pobreza tiene mayor porcentaje de calidad adecuada del patrón de consumo alimentario que el cuartil de mayor pobreza, lo contrario sucede en cuanto a la calidad inadecuada. Conclusiones: La calidad del patrón de consumo en general y por grupos de alimentos está asociada a la pobreza de acceso a los alimentos.

Palabras Clave: Alimentación, Pobreza, México.

\section{ABSTRACT}

Introduction: Food is the primary factor that affects health, the quality of the pattern of food consumption can be modified by poverty due to access to food. Objective: To estimate whether the quality of food is associated with poverty due to access to food in the population of northeastern Mexico. Material and methods: The database of the Nuevo León State Health and Nutrition Survey 2011/2012 was analyzed, which contains a probabilistic sample of 7,290 individuals. The quality variable of the food consumption pattern was constructed from the State Survey of Health and Nutrition Nuevo Leon 2011/2012 and the variable poverty for access to food from national registers. Nonparametric statistical tests were used. Results The highest percentage of the analyzed population is in the category of need for change in the quality of the pattern of food consumption, the lowest-poverty quartile has a higher percentage of adequate quality of the food consumption pattern than the highestpoverty quartile, the opposite happens in terms of inadequate quality. Conclusions: The quality of the consumption pattern in general and by food groups is associated with poor access to food.

Key words: Food, Poverty, Mexico 


\section{Introducción}

La alimentación es el principal factor en la salud, su efecto se puede potenciar al combinarse con otros factores tales como la actividad física, el estilo de vida, pobreza, cultura alimentaria. (Carcamo \& Mena, 2006)

Para caracterizar el determinante de la alimentación es necesario practicar encuestas poblacionales. En México, se realiza la Encuesta Nacional de Salud y Nutrición (ENSANUT) que tiene varias ediciones y son referente nacional. En Nuevo León se ha desarrollado por primera vez la EESNL- 2011 (2012), en ella se reportan diferentes determinantes sociales y, asimismo, la caracterización del patrón alimentario, el cual no difiere de los alimentos consumidos en el año 2000 (Ramos Peña, y otros, 2005). Sin embargo, dado que no es el espíritu de las encuestas poblacionales desarrollar el análisis de la alimentación, ésta sirve para realizar estudios de alimentación a la luz de los determinantes sociales u otras variables.

El análisis de las variables se realizó en una entidad federativa que se encuentra en el noreste de México, colinda con la frontera sur de Estados Unidos de Norteamérica. Tiene 5;157,780 habitantes (INEGI, 2017), 504,330 habitantes más que en el 2010 ( (INEGI, 2010), a pesar de que es una entidad que aporta un alto porcentaje al PIB nacional (INEGI, 2011) tiene población en pobreza por acceso a los alimentos; en la entidad federativa aumentó en 3,800 personas en pobreza por acceso a la alimentación.

El objetivo de este estudio es analizar si calidad de la alimentación está asociada a la pobreza por acceso a los alimentos en población de 5 a 59 años de la entidad federativa del noreste mexicano.

\section{Material y Métodos}

Se trata de un estudio descriptivo, transversal, analítico. Como fuente de información se utilizó la Encuesta Estatal de Salud y Nutrición (EESN-NL2011/2012) que se aplicó a la población de 0 a 69 años de Nuevo León cuya metodología de la encuesta ha sido publicada (Secretaría de Salud de Nuevo León; Universidad Autónoma de Nuevo León; Facultad de Salud Pública y Nutrición; Facultad de Medicina; Sistema para el Desarrollo Integral de la Familia del Estado de Nuevo León; Cáritas de Monterrey ABP, 2013).
Para el caso de este estudio, se analizaron a 7,293 que contiene la base de datos; como criterios de inclusión se tomaron en cuenta a individuos de 5 a 59 años (ya que fuera de esos rangos se utilizó otro instrumento de recolección de la información), ambos géneros, que tuvieran la información de los alimentos seleccionados.

\section{Alimentación}

Para la construcción de la variable calidad de alimentación en la población se utilizó el patrón de consumo alimentario derivando en la calidad del patrón de consumo alimentario, se estratificó en rangos mediante la propuesta de Norte-Navarro y Ortiz-Moncada (Norte \& Ortiz, 2011), se aceptaron los rangos de las categorías, grupos de alimentos y los puntajes de corte para estimar la calidad, ( $>$ de 80 "saludable", $>50-\leq 80$ "necesita cambios", $\leq 50$ "poco saludable") que sugieren. Para establecer lo que sería la CPCA por grupo de alimento, se utilizaron puntajes de corte ( $>$ de 8 "saludable", $>5$ $\leq 8$ "necesita cambios", $\leq 5$ "poco saludable"). Hemos decidido el nombre de calidad del patrón de consumo alimentario (CPCA) el cual se refiere a un índice de calidad de la dieta en relación a la variedad en el consumo de los grupos de alimentos y los nombres de las categorías se establecieron como adecuada, necesita cambios, inadecuada, ya que con la frecuencia de consumo de alimentos no es posible determinar si es o no saludable (Ramos Peña, Ramírez López, Salas García, Núñez Rocha, \& Villarreal Pérez, 2016).

\section{Pobreza por acceso a los alimentos}

Para la construcción de la variable pobreza por acceso a los alimentos, los municipios fueron estratificados en cuartiles de pobreza por acceso a los alimentos a partir del porcentaje de población con pobreza por acceso a los alimentos en los municipios derivado de los datos del Consejo Nacional de Evaluación de la Política Social (CONEVAL), en el primer cuartil están todos aquellos municipios que tienen el menor porcentaje de habitantes con este tipo de pobreza $(4.74 \%$ a $13.87 \%)$, segundo cuartil del $13.9 \%$ a $16.31 \%$, tercer cuartil del $16.67 \%$ al $20.32 \%$ y, el ultimo cuartil del $21.18 \%$ al $42.34 \%$, que es el de mayor porcentaje de población con este tipo de pobreza (CONEVAL, Medición de la pobreza: Resultados de pobreza por municipio 2010, s.f.) 
Para el análisis estadístico se utilizó la prueba no paramétrica de independencia de $\mathrm{Chi}^{2}$. El software utilizado fue el NCCS10.0 (2015).

\section{Resultados}

De la base de datos que consta de 7,290 individuos en este análisis no se incluyeron a los niños menores de cinco años (608 niños) y a los adultos mayores de 60 años y más $(1,474)$, asimismo, se excluyeron a 13 individuos por información incompleta.

Se analizaron a 5,195 individuos, el mayor porcentaje se encontró en los municipios que tienen mayor porcentaje de población en pobreza por acceso a los alimentos, así mismo, en lo referente a CPCA general (esto es, todos los grupos de alimentos, el mayor porcentaje necesita cambios) (ver tabla 1)

Tabla 1. Población por cuartiles de municipios con pobreza por acceso a los alimentos y CPCA

\begin{tabular}{lcc}
\hline & No. & $\%$ \\
\hline Cuartiles & & \\
Q1 & 884 & 17.0 \\
Q2 & 1375 & 26.5 \\
Q3 & 1305 & 25.1 \\
Q4 & 1631 & 31.4 \\
& & \\
CPCA general & & \\
Adecuada & 96 & 1.8 \\
Necesita Cambios & 4359 & 83.9 \\
Inadecuada & 740 & 14.2 \\
\hline
\end{tabular}

Fuente: EESNL-2011/2012

$n=5195$

En cuanto a la CPCA en general, el $1.84 \%$ tiene CPCA adecuada, $83.9 \%$ se ubica en necesidad de cambios y el $14.2 \%$ en inadecuada. Al estratificar la CPCA por cuartiles de municipios según el porcentaje de pobreza por acceso a los alimentos, el menor porcentaje de población en la categoría de CPCA adecuada lo tiene el cuartil 4 (Q4) respecto al cuartil 1; En la categoría de CPCA con necesidad de cambio, el menor porcentaje se encuentra en el cuartil 1 y el mayor en el cuartil 4, esta distribución sucede de manera similar en la categoría de CPCA inadecuada. Se observa una tendencia inversa en los porcentajes de CPCA adecuada en relación a pobreza por acceso a los alimentos y en las otras dos categorías de CPCA (necesita cambios e inadecuada) una tendencia directa. Se ha encontrado asociación en la población de estudio entre la CPCA y la pobreza por acceso a los alimentos $(\mathrm{p}>$.05) (ver tabla $2)$.

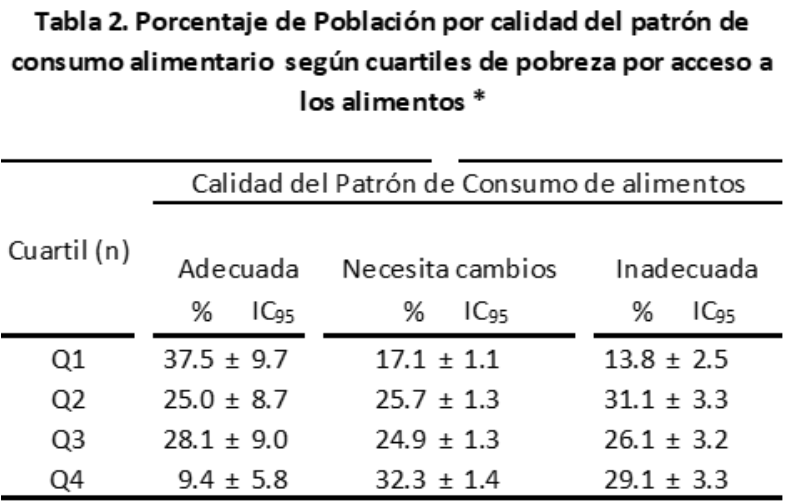

Fuente: EESNL-2011/2012

$* \mathrm{Ji}^{2}=\mathrm{P}<.05$

Respecto a la CPCA por grupos de alimentos y los cuartiles de pobreza por acceso a los alimentos, a excepción del grupo de los dulces, las diferencias marcadas se encuentran en las categorías de CPCA inadecuada y adecuada, siendo significativas en los cereales, verduras, frutas, lácteos y refrescos. En el grupo de carnes, no se encontraron individuos que tuvieran CPCA adecuada. En todos los grupos de alimentos, la CPCA se encuentra asociada a la pobreza por acceso a los alimentos ( $p>.05)$ (ver tabla $3)$. 
Tabla 3. Porcentaje de Población por calidad del patrón de consumo alimentario y grupo de alimentos según cuartiles de pobreza por acceso a los alimentos.

\begin{tabular}{|c|c|c|c|}
\hline \multirow{3}{*}{ Cuartiles } & \multicolumn{3}{|c|}{ Calidad del Patrón de Consumo de alimentos } \\
\hline & Adecuado & Necesita & Inadecuado \\
\hline & $\% \quad I C_{95}$ & $\% \quad I C_{95}$ & $\% \quad I C_{95}$ \\
\hline Cereales* & 4415 & 652 & 128 \\
\hline Q1 & $16.3 \pm 1.1$ & $23.6 \pm 3.3$ & $9.4 \pm 5.1$ \\
\hline Q2 & $25.8 \pm 1.3$ & $29.8 \pm 3.5$ & $31.3 \pm 8.0$ \\
\hline Q3 & $24.4 \pm 1.3$ & $28.1 \pm 3.5$ & $33.6 \pm 8.2$ \\
\hline Q4 & $33.5 \pm 1.4$ & $18.6 \pm 3.0$ & $25.8 \pm 7.6$ \\
\hline Verduras* & 2806 & 1509 & 880 \\
\hline Q1 & $15.5 \pm 1.3$ & $21.1 \pm 2.1$ & $14.9 \pm 2.4$ \\
\hline Q2 & $26.3 \pm 1.6$ & $27.1 \pm 2.2$ & $25.8 \pm 2.9$ \\
\hline Q3 & $24.0 \pm 1.6$ & $23.9 \pm 2.2$ & $30.8 \pm 3.1$ \\
\hline Q4 & $34.2 \pm 1.8$ & $27.9 \pm 2.3$ & $28.5 \pm 3.0$ \\
\hline Frutas* & 504 & 2620 & 2071 \\
\hline Q1 & $19.8 \pm 3.5$ & $19.2 \pm 1.5$ & $13.6 \pm 1.5$ \\
\hline Q2 & $29.0 \pm 4.0$ & $27.1 \pm 1.7$ & $25.0 \pm 1.9$ \\
\hline Q3 & $29.8 \pm 4.0$ & $25.1 \pm 1.7$ & $24.0 \pm 1.8$ \\
\hline Q4 & $21.4 \pm 3.6$ & $28.6 \pm 1.7$ & $37.3 \pm 2.1$ \\
\hline Lacteos* & 2164 & 1809 & 1222 \\
\hline Q1 & $20.9 \pm 1.7$ & $17.6 \pm 1.8$ & $9.3 \pm 1.6$ \\
\hline Q2 & $25.5 \pm 1.8$ & $28.4 \pm 2.1$ & $25.4 \pm 2.4$ \\
\hline Q3 & $28.8 \pm 1.9$ & $23.7 \pm 2.0$ & $20.7 \pm 2.3$ \\
\hline Q4 & $24.8 \pm 1.8$ & $30.3 \pm 2.1$ & $44.6 \pm 2.8$ \\
\hline Carnes* & 0 & 5024 & 171 \\
\hline Q1 & $0.0 \pm 0.0$ & $17.2 \pm 1.0$ & $12.3 \pm 0.6$ \\
\hline Q2 & $0.0 \pm 0.0$ & $26.8 \pm 1.2$ & $17.0 \pm 2.0$ \\
\hline Q3 & $0.0 \pm 0.0$ & $25.6 \pm 1.2$ & $12.3 \pm 1.8$ \\
\hline Q4 & $0.0 \pm 0.0$ & $30.5 \pm 1.3$ & $58.5 \pm 0.9$ \\
\hline Leguminosas* & 1115 & 1008 & 3072 \\
\hline Q1 & $24.9 \pm 2.5$ & $17.7 \pm 2.4$ & $13.9 \pm 1.2$ \\
\hline Q2 & $28.6 \pm 2.7$ & $28.5 \pm 2.8$ & $25.0 \pm 1.5$ \\
\hline Q3 & $30.7 \pm 2.7$ & $27.2 \pm 2.7$ & $22.4 \pm 1.5$ \\
\hline Q4 & $15.8 \pm 2.1$ & $26.7 \pm 2.7$ & $38.6 \pm 1.7$ \\
\hline Embutidos* & 1467 & 3457 & 1467 \\
\hline Q1 & $9.5 \pm 1.5$ & $19.9 \pm 1.3$ & $20.7 \pm 4.8$ \\
\hline Q2 & $16.6 \pm 1.9$ & $30.3 \pm 1.5$ & $31.0 \pm 5.5$ \\
\hline Q3 & $17.8 \pm 2.0$ & $27.6 \pm 1.5$ & $33.6 \pm 5.6$ \\
\hline Q4 & $56.0 \pm 2.5$ & $22.2 \pm 1.4$ & $14.8 \pm 4.2$ \\
\hline Dulces* & 3475 & 1469 & 251 \\
\hline Q1 & $15.5 \pm 1.2$ & $18.9 \pm 2.0$ & $26.7 \pm 5.5$ \\
\hline Q2 & $25.2 \pm 1.4$ & $30.8 \pm 2.4$ & $19.5 \pm 4.9$ \\
\hline Q3 & $25.1 \pm 1.4$ & $24.4 \pm 2.2$ & $29.9 \pm 5.7$ \\
\hline Q4 & $34.2 \pm 1.6$ & $25.9 \pm 2.2$ & $23.9 \pm 5.3$ \\
\hline Refrescos* & 1010 & 978 & 3207 \\
\hline Q1 & $17.9 \pm 2.4$ & $17.5 \pm 2.4$ & $16.6 \pm 1.3$ \\
\hline Q2 & $23.1 \pm 2.6$ & $27.3 \pm 2.8$ & $27.3 \pm 1.5$ \\
\hline Q3 & $24.9 \pm 2.7$ & $29.6 \pm 2.9$ & $23.9 \pm 1.5$ \\
\hline Q4 & $34.2 \pm 2.9$ & $25.7 \pm 2.7$ & $32.3 \pm 1.6$ \\
\hline
\end{tabular}

Fuente: EESNL-2011/2012

$* \mathrm{Ji}^{2}=\mathrm{P}<05$ dentro del grupo de alimentos

\section{Discusión}

En ésta investigación, encontramos que la calidad de la alimentación en población del noreste de México, medida a través de la CPCA, se encuentra asociada a la pobreza por acceso a los alimentos, tanto de manera general como en los grupos de los alimentos.

La salud es una variable multicausal, se ve influida por determinantes sociales (desde estructurales hasta proximales), dentro de los proximales, la alimentación es uno de los principales, si la alimentación no se ejerce de manera correcta, puede tener efecto nocivo sobre la salud. En la alimentación, la población puede actuar para coadyuvar en la prevención de enfermedades secundarias a la mala alimentación, como son las enfermedades crónicas no trasmisibles, entre otras, la diabetes, hipertensión y obesidad (Ramos Peña, Ramírez López, Salas García, Núñez Rocha, \& Villarreal Pérez, 2016). La asociación entre enfermedades crónicas no trasmisibles, como la diabetes, hipertensión arterial (Del Ángel \& Villagómez, 2014), sobrepeso y obesidad con la alimentación ha sido estimada en varias investigaciones (Casals, Suárez, Estébanez, Aguilar, \& Jiménez, 2017; González, y otros, 2015; Ortega, Jiménez, Perea, Cuadrado, \& López, 2016; Muñoz \& Carranza, 2010; Reséndiz, Hernández, Sierra, \& Torres, 2015) lo que demuestra la aplicabilidad de la CPCA grupos poblacionales para prevenir las enfermedades referidas en los diferentes niveles de los modelos de prevención, asimismo, se ha encontrado mayor prevalencia de ENT en niveles socioeconómicos bajos que en medios y alto . La ESSNL 2011 (2012), estimó la prevalencia de diabetes mellitus tipo 2 en adultos mayores de 20 a 59 años en $14.9 \%$, hipertensión arterial en el $16.9 \%$, obesidad y sobrepeso en el $71.7 \%$. En el grupo de 10 a 19 años, la obesidad y el sobrepeso pueden estar entre $33.9 \%$ y el $39.3 \%$, otro dato que se debe agregar al panorama de salud en Nuevo León, es la prevalencia de síndrome metabólico entre la población de 16 años o más es de $45.3 \%$ en hombres y 59.4\% en mujeres del 54.9\% (Salas, y otros, 2014), a este panorama epidemiológico, podremos sumar que los resultados de este estudio muestran que gran porcentaje de la población analizada tiene calidad no adecuada de la alimentación, $80 \%$ tiene necesidad de cambio en la CPCA y en algunos estratos el porcentaje puede rebasar al $90 \%$ cuando se agrega el porcentaje que tiene CPCA inadecuada. 
En la atención de la alimentación a poblaciones existe la estrategia de la orientación alimentaria que se aplica de manera general, pero también, las orientaciones abarcan las necesidades por grupos de edad y sexo, sin embargo, es posible que la población que habita en municipios con algún grado de condición de pobreza por acceso a los alimentos no tenga la posibilidad de seguir las indicaciones mencionadas por los orientadores en nutrición y alimentación. Es necesario determinar si la CPCA en esta población, que está asociada a la edad y al sexo (Ramos Peña, Ramírez López, Salas García, Núñez Rocha, \& Villarreal Pérez, 2016), se mantiene en presencia de la pobreza por acceso a los alimentos, pues de no ser así, se debe atender la pobreza por acceso a los alimentos para fortalecer la a la orientación alimentaria ya que la población no tendría la variedad necesaria de alimentos en razón de edad y sexo.

El estudio tiene como fortaleza el análisis en una muestra probabilística de población del noreste mexicano, pero tiene como limitante el hecho de que se trata de una frecuencia alimentaria que no es semicuantitativa y que analiza solo la calidad del patrón de consumo por variedad y grupos de alimentos y no el índice de alimentación saludable.

\section{Conclusiones}

El estudio, en esta población del noreste mexicano, aporta evidencia que las variables calidad de la alimentación y la pobreza por acceso a los alimentos están asociadas, asimismo, demuestra que existe menor porcentaje de población con CPCA adecuada en municipios con mayor pobreza por acceso a los alimentos que en los de menor pobreza, lo contrario sucede en cuanto a la CPCA inadecuada. Los resultados pueden ser utilizados en estrategias y acciones de orientación alimentaria en los diferentes estratos de pobreza por acceso a los alimentos a fin de prevenir en todos los niveles del proceso salud enfermedad que se puedan encontrar asociados a la alimentación.

\section{Bibliografía}

Casals, C., Suárez, -C., Estébanez, C. F., Aguilar, T. M., \& Jimenez, A. M.-S. (2017). Relación entre la calidad de vida, actividad física, alimentación y control glucémico con la sarcopenia de adultos mayores con diabetes mellitus tipo 2. Nutrición Hospitalaria, 34(5), 1198-1204. doi:10.20960/nh.1070
CONAPO. (2012, junio). Consejo Nacional de Población. Retrieved julio 2, 2015, from http://www.conapo.gob.mx/es/CONAPO/Capitulo_1 Marginacion_Urbana_2010

CONAPO. (2012, julio 27). Consejo Nacional ded Población. Retrieved julio 2, 2015, from http://www.conapo.gob.mx/work/models/CONAPO/i ndices margina/mf2010/CapitulosPDF/Anexo\%20B3 .pdf

CONAPO. (2012). Índice de Marginación por Localidad 2010. (G. d. México, Ed.) Retrieved Junio 27, 2015, from Consejo Nacional de Población: http://www.conapo.gob.mx/en/CONAPO/Indice de Marginacion_por_Localidad_2010

CONEVAL. (2012). Informe de Pobreza en México, 2010: el país, los estados y sus municipios, . México.

CONEVAL. (n.d.). CONEVAL. Retrieved enero 13, 2016, from

http://www.coneval.org.mx/Medicion/MP/Paginas/An exo-estad\%C3\%ADstico-municipal-2010.aspx

Darmon, N. D. (2008). Does social class predict diet quality? Am J Clin Nutr, 87(5), 1107-17.

Del Angel, -P. A., \& Villagómez, -C. J. (2014). Alimentación, salud y pobreza en áreas marginadas urbanas: caso Veracrúz-Boca del Río, veracruz. Estudios Sociales Revista de Investigación Científica, 22(44), 9-35.

González, S. R., Llapur, M. R., Diaz, C. M., Lila, C. M., Yee, L. E., \& Pérez, B. D. (2015). Estilos de vida, hipertensión arterial y obesidad en adolescentes. revista Cubana de Pediatría, 273-284(3), 273-284.

Hintze, J. (2015). NCSS 10, Statistical Software. Kaysville, Utah, USA. Retrieved from ncss.com/software/ncss

INEGI. (2010). INEGI. (G. d. México, Editor) Retrieved junio 2015, 2015, from http://www.inegi.org.mx/lib/olap/consulta/general ve r4/MDXQueryDatos.asp?proy=cpv10_pt

INEGI. (2011). INEGI. Retrieved 2015, from http://cuentame.inegi.org.mx/monografias/informacio $\mathrm{n} / \mathrm{NL} /$ Economia/default.aspx?tema=ME\&e $=19$

INEGI, I. N. (2017). Anuario Estadístico y geográfico por entidad federativa 2017. Aguascalientes. 
Muñoz, C. J., Córdova, H. J., \& Valle, L. D. (2015). El indice de alimentación saludable de estudiantes de nuevo ingreso a una universidad de México. Nutrición Hospitalaria, 31(4), 1582-1588.

Muñoz, -I. A., \& Carranza, -M. J. (2010). Perfila Alimentario de una población rural de Michoacán y su asociación con obesidad, diabetes e hipertensión. Medicina Interna de México, 24-30.

Norte, N. A., \& Ortiz, M. R. (2011). Calidad de la dieta española según el ínidice de alimentacin saludable. Nutrición Hospitalaria, 26(2), 330-336. doi:DOI:10.3305/nh.2011.26.2.4630

OPS. (2013, octubre 3). 53o. Consejo Directivo, 66a. sesión del Comité Regional de la OMS para las Américas. Washington: OMS. Retrieved junio 30, 2015, from http://paho.org

Ortega, A. R., Jimenez, O. A., Perea, S. J., Cuadrado, S. E., \& López, -S. A. (2016). Pautas nutricionales en prevención y control de la hipertensión arterial. Nutrición Hospitalaria, 33(Suppl 4), 53-58. doi:10.20960/nh.347

Ramos Peña, E. G., Ramírez López, E., Salas García, R., Núñez Rocha, G. M., \& Villarreal Pérez, J. Z. (2016, Mzo 30). Calidad del patrón de consumo alimentario en población del noreste de México. Revista de Salud Pública y Nutrición, 15(1), 8-15. Retrieved Mayo 2018, from www.respyn.uanl.mx

Ramos Peña, E., Valdés Lozano, C., Cantú Martínez, C., Salinas García, G., Y.E., G. C., \& Salazar Garza, G. .. (2005, Dic). Patrón de consumo alimentario familiar en Nuevo León (México). Revista de Salud Pública y Nutrición, 6(4), 1-29. Retrieved ene 15, 2018, from www.faspyn.respyn.uanl.mx

Ramos, P. E., Castro, S. A., De la Garza, C. Y., Berrún, C. L., \& González, R. L. (2010, Julio-Septiembre). Propuesta de una Política Social Alimentaria Nutricional para Nuevo León. Revista de Salud Pública y Nutrición, 11(3). Retrieved julio 3, 2015, from http://www.respyn.uanl.mx/xi/3/ensayos/politica_ali mentaria.htm

Reséndiz, B. A., Hernández, A. S., Sierra, M. M., \& Torres, T. M. (2015). Hábitos de alimentación de pacientes con obesidad severa. Nutrición Hospitalaria, 31(2), 672681. Retrieved from 10.3305/nh.2015.31.2.7692

Salas, R., Del Mar, B. M., Ramos, E., Villarreal, J. Z., Pons, A., Tur, J. A., \& Sureda, A. (2014, Agosto). Metabolic Syndrome Prevalence among Northern. Plos One, 9(8), 1-9. Retrieved from www.plosone.org
Schwingshackl, L., \& Hoffmann, G. (2015, mayo). Diet Quality as Assessed by the Healthy Eating Index, the Alternate Healthy Eating Index, the Dietary Approaches to Stop Hypertension Score, and Health Outcomes: A Systematic Review and Meta-Analysis of Cohort Studies. Journal of the Academy of Nutrition \& Dietetics, 115(5), 780-800. Retrieved julio 3, 2015, from

http://web.b.ebscohost.com.remoto.dgb.uanl.mx/ehost /detail/detail?vid=23\&sid=8899312a-affd-4634-b27e28ee0b9bdcaa\%40sessionmgr113\&hid $=123 \&$ bdata $=\mathrm{J}$ kF1dGhUeXB1PWlwLHVybCx1aWQsY29va21lJmxh bmc9ZXMmc210ZT1laG9zdC1saXZl\#db=a9h\&AN= 102189890

Secretaria de Gobernación. (2013). NORMA Oficial Mexicana NOM-043-SSA2-2012, Servicios básicos de salud. Promoción y educación para la salud en materia alimentaria. Criterios para brindar orientación. México: Gobierno de México. Retrieved julio 3, 2015, from

http://www.dof.gob.mx/nota_detalle.php? codigo $=528$ $\underline{5372 \& \text { fecha }=22 / 01 / 2013}$

Secretaria de Salud. (2013). Estrategia Nacional para la Prevención y el Control del Sobrepeso, la Obesidad y la Diabetes. México: IEPSA. Retrieved 2015

Secretaría de Salud. (2013). Estrategia Nacional para la Prevención y el Control del Sobrepeso, la Obesidad y la Diabetes . México: IEPSA.

Secretaría de Salud de Nuevo León; Universidad Autónoma de Nuevo León; Facultad de Salud Pública y Nutrición; Facultad de Medicina; Sistema para el Desarrollo Integral de la Familia del Estado de Nuevo León; Cáritas de Monterrey ABP. (2013). Encuesta Estatal de Salud y Nutrición- Nuevo León 2011/2012 (EESNL-NL 2011/2O12). Monterrey. Retrieved enero 2018 , from http://journals.plos.org/plosone/article/file?id=info\%3 Adoi/10.1371/journal.pone.0155994.s001\&type=supp lementary

Secretaría de Salud Nuevo León. (2014). Estrategia Estatal para la Prevención y el Control del Sobrepeso, la Obesidad y la Diabetes en Nuevo León. Monterrey: Secretaria de Salud Nuevo León.

Secretaria de Salud; Universidad Autónoma de Nuevo León; Facultad de Salud Pública; Facultad de Medicina; Sistema para el Desarrollo Integral de la Familia; Cáritas de Monterrey ABP; Secretaria de Educación de Nuevo León. (2012). Encuesta Estatal de Salud y Nutrición 2011/2012. Monterrey: Gobierno de Nuevo León. 
Tande, D., Magel, R., \& Strand, B. (2010). Healthy Eating Index and abdominal obesity. Public Health Nutrition, 13(2), 208-214. Retrieved julio 2, 2015, from http://web.b.ebscohost.com.remoto.dgb.uanl.mx/ehost /detail/detail?vid=18\&sid=8899312a-affd-4634-b27e28ee0b9bdcaa\%40sessionmgr113\&hid $=123 \&$ bdata $=\mathrm{J}$ kF1dGhUeXB1PWlwLHVybCx1aWQsY29va211Jmxh bmc9ZXMmc210ZT1laG9zdC1saXZl\#db=a9h\&AN= $\underline{47748124}$ 